Wefelmeyer, F. Die Sehnsucht nach Transzendenz

\title{
Die Sehnsucht nach Transzendenz Zu ihrer Bedeutung im Werk von Botho Strauß
}

[Nostalgia for transcendence: on its meaning in B. Strauß' work]

Fritz Wefelmeyer ${ }^{1}$

\begin{abstract}
In his writing Botho Strauß deals with the question of a longing for transcendence over a number of years and from a number of different perspectives. The article firstly considers the way in which social theories and world views which Strauß originally followed have led this longing in the wrong direction and ignored the true role of technology. Strauß' own analysis, which incorporates considerations in particular from the natural sciences, fails to take adequate account of creative activity. Another perspective emerges from Strauß' representation of the unhappy consciousness of the modern individual and of the attempts made to transcend this. Finally, Strauß' own attempt to achieve transcendence through art is examined. The article draws attention here again to the under-developed role of creative activity in his work.
\end{abstract}

Keywords: Social theories, unhappy consciousness, technology, work of art, creativity

Zusammenfassung: Botho Strauß hat die Frage der Sehnsucht nach Transzendenz von verschiedenen Gesichtspunkten aus immer wieder in seinem Werk behandelt. Da ist zunächst der Punkt, wie Gesellschaftstheorien und Weltbilder, denen er ursprünglich selbst gefolgt ist, diese Sehnsucht in eine falsche Richtung gelenkt und die wirkliche Rolle von Technologie unbehandelt gelassen haben. Strauß' eigene Analyse, die Überlegungen vor allem aus den Naturwissenschaften aufnimmt, vernachlässigt aber die Bedeutung schöpferischer Aktivität. Ein weiterer Gesichtspunkt ergibt sich aus Strauß' Darstellung des unglücklichen Bewusstseins des modernen Individuums und den Versuchen, dieses zu transzendieren. In einem letzten Punkt wird dann Strauß' eigener Versuch erörtert, im Kunstwerk Transzendenz zu schaffen. Der Artikel macht hier erneut auf die unterbelichtete Rolle schöpferischer Aktivität aufmerksam.

Stichwörter: Gesellschaftstheorien, unglückliches Bewusstsein, Technik, Kunstwerk, Schöpfung

${ }^{1}$ Dr phil., University of Sunderland, Department of Culture. Email: fritz.wefelmeyer@sunderland.ac.uk

Pandaemonium, São Paulo, v. 16, n. 21, Jun/2013, p. 1-24 www.fflch.usp.br/dlm/alemao/pandaemoniumgermanicum 
Wefelmeyer, F. Die Sehnsucht nach Transzendenz

\section{Einleitung}

Die vorliegende Untersuchung möchte auf einige Schwierigkeiten aufmerksam machen, die sich in der Behandlung des im Aufsatztitel genannten Themas im Werk von Botho Strauß ergeben. Dieser hat das Thema in enger Verbindung mit dem Verlust traditioneller Orientierungen und wissenschaftlich-philosophischer, aber auch religiöser Weltbilder gesehen; seine Bühnenstücke zeugen davon. Dieser Verlust war aber selbstverständlich nicht einfach hinzunehmen und das essayistische Werk macht bis in die Gegenwart hinein deutlich, in welch weit ausgreifender Weise Botho Strauß sich mit neuen Theorie- und Weltbildentwürfen beschäftigt hat. Insofern steht seine eigene Lebensgeschichte für das Bedürfnis nach Transzendenz ein. Nirgendwo ist dies deutlicher als in seinem Buch Beginnlosigkeit (1992). An gleicher Stelle aber bekennt der Autor, dass die Proliferation neuer Weltbilder, sei es in der Neuroscience, in der Genetik, in der Astrophysik, die auf den rasanten Zusammenbruch der vorausgegangenen Bilder folgt, von dem gleichen Schicksal bedroht ist, das die Vorgänger ereilt hat (ebd. 14). Freilich liegt in dem Ende solcher Weltbilder auch die Chance der Befreiung, denn diese engen den Horizont oft ein, statt ihn zu erweitern. Im Falle von Strauß ist die Befreiung von einem solchen Weltbild, wie es die kritisch-marxistische Gesellschaftstheorie geliefert hatte, einst entscheidend gewesen, sie hat seine weitere Behandlung des Themas von Sehnsucht und Transzendenz entscheidend geprägt. Sie hat aber auch die Weichen gestellt, die zu Schwierigkeiten geführt haben.

Um diese Schwierigkeiten aufzuspüren, sollen im weiteren einige Unterscheidungen getroffen werden. Ich spreche von Sehnsucht nach Transzendenz und nicht, wie zum Beispiel Dorothee Fuß, vom “Bedürfnis nach Heil” im Werk von Botho Strauß (FUß 2001). Der Begriff Heil ist im Werk von Strauß stärker auf eschatologische und geschichtsphilosophische Bezüge hin angelegt als der Begriff Transzendenz. Von heilsgeschichtlichen Erwartungen will Strauß sich aber in seiner eigenen Position gerade absetzen. Der Begriff Transzendenz steht solchen Erwartungen neutraler gegenüber. Von Sehnsucht nach Transzendenz lässt sich weiterhin im Werk von Botho Strauß in mindestens dreifacher Hinsicht sprechen. Einmal ist diese Sehnsucht der Ausdruck einer 
intellektuellen und emotionalen Befindlichkeit, die mit einer bestimmten gesellschaftskritischen Philosophie aufgekommen ist. Spätestens seit dem Ende der sechziger Jahre hat diese Befindlichkeit, wie übrigens auch die sie tragende Philosophie, weite Aufnahme und Verbreitung im Bereich öffentlichkeitsbewusster und sozialwissenschaftlich interessierter Kreise gefunden. Die linksorientierte kritische Intelligenz bildete hier den idealen Nährboden dieser hochverfeinerten, an den gesellschaftlichen Verhältnissen leidenden Befindlichkeit.

Für Strauß handelt es sich dabei, um das gleich vorwegzunehmen, um ein profanisiertes Transzendenzverlangen. Dieses Verlangen oder Sehnsucht - zu ihrer Kennzeichnung wähle ich hier und im folgenden eine physiognomische Schreibweise, wie sie aus den Texten von Strauß selbst bekannt ist - ist der mittlerweile trocken gewordene Reiseproviant, den ein christliches Zeitalter einst für die Reise durch das weltliche Jammertal bereitgestellt hatte. Im 20. Jahrhundert, unter den Bedingungen einer emanzipativen Vernunft- und Aufklärungsfrömmelei, wird diese Sehnsucht, laut Strauß, zu einem Betäubungsmittel und Gift, die das kraftvolle Ergreifen und die freudige Feier des Lebens lähmen. Strauß spricht gar vom Verrat am Leben. Man könnte auch sagen, dass die Träger dieses Verlangens sich in Reaktion auf die jüngere deutsche Geschichte zwischen 1933 und 1945 und deren Fortwirken, aber auch in Reaktion auf politische Veränderungen (Notstandsgesetze, Vietnamkrieg) auf eine Form der Rationalität - Stichwort: Kapitalismuskritik - festgelegt hatten, die sich als Fessel erwies. Das Buch Paare Passanten (1981) bot bereits erste Beobachtungen und Reflexionen dazu an.

Die zweite Hinsicht, in der man von dieser besonderen Sehnsucht sprechen kann, hängt durchaus mit der ersten Hinsicht zusammen. Wie nämlich diese zuerst genannte Sehnsucht mit dem modernen gesellschaftlichen Leben verbunden ist, so auch diese andere Form der Sehnsucht. Sie ist der Reflex des Einzelnen auf gesellschaftliche Veränderungen. Diese Sehnsucht zeigt sich als die verquere Hoffnungsspur auf der individuell beleuchteten Großaufnahme, die das miserable und verfehlte Leben aller darbietet. Der Glimmer einer vagen Verheißung ist sie, der aus einer lebensgeschichtlichen Misere aufsteigt, die zwischen neudeutscher Abgeklärtheit, Dauerberieselung durch die mediale Öffentlichkeit, 
Wefelmeyer, F. Die Sehnsucht nach Transzendenz

Statusängsten und Gefühlsvermischung sich abspielt. Verzweifelt sucht diese Sehnsucht sich aus dem Stimmungsmix und der permanenten Stimmungsreflexion herauszuwinden auf das "ganz andere" hin. Ist diese Suchbewegung bereits erstorben, so kommt doch bei dem Leser oder Zuschauer bisweilen der Wunsch auf, angeregt durch Strauß' Darstellung, den literarischen Stellvertretern des neudeutschen Menschen aus ihrem täglichen Einerlei einen Ausweg zu verschaffen. Und eines Ausweges bedürfen diese literarisehen Figuren auch, wenn nach den hochfliegenden Aspirationen, die sie mit Partnern, Liebhabern, Freunden, mit Karrieren, Lebensentwürfen und Erfolgskonzepten gehabt haben, nur die Ausnüchterung und der existentielle Stimmungskater übrigbleibt.

Wo tritt diese Sehnsucht auf? Sie geht quer durch alle Schichten, Gruppen und Gehaltsklassen. Sie tritt im Turnvereinsmitglied so gut auf wie im Probanden einer Yoghurttestgruppe. Das Individuum, das, adrett gekleidet, die Flughafenkontrolle über sich ergehen lässt, wird von ihr ebenso angefallen wie der Nackte unter dem MRI-Scanner oder vor der Digitalkamera. Diese Sehnsucht drückt aus, was am Ende aller gescheiterten Selbstverwirklichung das Individuum sich noch erhoffen kann - wenn ihm die Desillusionierung das noch erlaubt! Ich werde mich allerdings mit dieser Form der Sehnsucht nicht sehr ausführlich auseinandersetzen, da sie von Strauß' Stücken und Prosaarbeiten her bereits bekannter als die anderen Formen sein dürfte. Die wissenschaftliche Sekundärliteratur hat sich ihr ja auch bereits schon unter vielfältigen Titeln gewidmet (vgl. NOWAK 2006).

Natürlich können diese beiden Formen der Sehnsucht kombiniert auftreten. Eine enge Verwandtschaft besteht auch zu der dritten Form der Sehnsucht, diese ist in manchem sogar ein Kind der beiden ersten, obwohl Strauß die Zeugenschaft dieser beiden nicht gern zugeben wird. Das Kind soll eben, um im Bild zu bleiben, von besseren Eltern abstammen. Handelt es sich also bei den beiden ersten Sehnsüchten um solche, die Strauß selbst diagnostiziert und seziert hat, vornehmlich wohl an Objekten, die zum Zeitpunkt der Diagnose das eigene Selbst nicht einschlossen, so ist die dritte Sehnsucht ganz die eigene Sehnsucht des Autors. Zwar nicht als solche benannt, aber für den Leser doch klar ersichtlich. Wir folgen also im weiteren bei der Analyse der beiden ersten Sehnsüchte dem Blick des Autors, während bei der letzten Sehnsucht der Blick auf den Autor selbst fällt: 
Wefelmeyer, F. Die Sehnsucht nach Transzendenz

was ist von den Aufschwüngen ins Transzendente zu halten, die der Diagnosekünstler selbst unternimmt? Glanzvolle Trapezakte oder wieder einmal, um einen alten Titel zu zitieren, Artisten in der Zirkuskuppel, ratlos (KLUGE 1968)?

\section{Falsche Weltbilder, Technik und Schöpfuns}

Will man die erste Form der Sehnsucht untersuchen, so ist es nützlich, zunächst einmal einen Blick auf die intellektuelle Geschichte des Autors Botho Strauß zu werfen. Dass sich der Autor nämlich in den achtziger Jahren so radikal von linken Transzendenzwünschen abgesetzt hatte, musste den erstaunen, der den Autor seit seinen ersten Arbeiten kannte. War er nicht damals gerade eben noch Kritiker bei der Zeitschrift Theater heute gewesen und hatte dort Artikel ganz im Sinne einer linken Sensibilität von '68 geschrieben (STRAUß 1987a)? Und war er dann nicht Mitarbeiter bei der Schaubühne gewesen, also bei jenem Theater, das der Fraktionsvorsitzende der CDU einst der kommunistischen Unterwanderung verdächtigt hatte? Und hatte er nicht selbst den großen Einfluss von Gesellschaftskritkern wie Theodor W. Adorno und Ernst Bloch auf sein Werk zugegeben (vgl. Richter 2010: 56-65)? In einem Interview aus dem Jahre 1980 sagt Strauß: "In meiner intellektuellen Erziehung hat die dialektische Schule eine große Rolle gespielt. [...] Man las alles von Benjamin und verschaffte sich mit einem Zitat das entsprechende Fluidum. Aus dieser Schulung bin ich nie herausgetreten und werde da wahrscheinlich auch nie herauskommen." (HAGE 1987: 199).

Doch es kam anders. Ein Satz in Klammern, also ein Satz, der mit einem gewissen Vorbehalt verbunden war, brachte damals die Wende. Geschrieben stand dieser Satz in Strauß' Paare Passanten, das einige Monate vor dem Regierungseintritt jener Partei, der CDU/CSU, erschein, die dann die politische Wende eingeleitete. Der Satz, im Original in Klammern stehend, lautete: “(Ohne Dialektik denken wir auf Anhieb dümmer; aber es muss sein: ohne sie!)” (STRAUß 1981: 115). Der rätselhafte Satz konnte damals nicht entschlüsselt werden. Man wusste nur, in welchen Kontext man ihn zu stellen hatte (Vgl. BAUER 2008: 98-102). Der dem eingeklammerten Satz unmittelbar vorausgehende Text 
Wefelmeyer, F. Die Sehnsucht nach Transzendenz

lautete: "Heimat kommt auf (die doch keine Bleibe war), wenn ich in den 'Minima Moralia' wieder lese. Wie gewissenhaft und prunkend gedacht wurde, noch zu meiner Zeit! Es ist, als seien seither mehrere Generationen vergangen.” (STRAUß 1981: 115).

In einem späteren Essay-Band hatte sich der Autor dann erkenntlicher ausgedrückt.

In Niemand anderes - wie schon Paare Passanten ein Verschnitt von akribisch genauen Einzelbeobachtungen im gesellschaftlichen Kräftefeld, poetisch literarischen Ausflügen ins Imaginäre und kurzen Diskursen und Reflexionen - heißt es dann:

[...] verwerflicher aber als die Urteile, welche die intime Überempfindlichkeit fällt, sind die, die sich gesellschaftskritischer Überheblichkeit verdanken. Grausam, totalitär, ein großer Geistesschaden ist zum Beispiel der Gedankengang vom allgemein beschädigten Leben, das auch im kleinen nur Beschädigtes zulässt. So auswegslos kann nur ein Gedankengang selber sein, niemals das offene Leben. (Strauß 1987: 202)

Und deutlicher noch, unmittelbar im Anschluss daran:

Erst die Befreiung von der Ananke-Idee, dass die jeweils ,bestehende' Ordnung unser tägliches Leben - etwas übergänglich Schlechtes sei; die Befreiung von jeder Art gesellschaftlichen Jenseits, von politisierter Erwartungsmetaphysik, die jeden täglichen Gang mit unerfülltem Dasein beschwert - erst also die konsequente Restverwirklichung der Welt wird die wahre und endliche Aufklärung voranbringen. Das Jenseits zurïck an seinen Platz! (ebd.)

Hier fehlen alle Klammern und es ist klar, dass eindeutig Adorno gemeint ist mit seinen Reflexionen aus dem beschädigten Leben, also die Minima Moralia mit ihrem bekannten Diktum, dass es kein wahres Leben im falschen geben könne (vgl. REUS 2006: 206-228). Mögen andere Stellen in dem Buch Niemand anderes noch eine ästhetische Lektüre nahelegen, also eine Lektüre, die den Text nicht für eine politische oder philosophische Überzeugung des Autors hält, sondern als Äußerung eines Erzählers oder einer Figur versteht, äußerlich etwa angedeutet durch den Gebrauch von Anführungszeichen und Inquitformeln, so zeigt diese Textstelle an, auch durch ihren näheren Kontext, dass sie, wie auch immer verwaschen, einem argumentativen Diskurs angehört.

Pandaemonium, São Paulo, v. 16, n. 21, Jun/2013, p. 1-24 www.fflch.usp.br/dlm/alemao/pandaemoniumgermanicum 
Wefelmeyer, F. Die Sehnsucht nach Transzendenz

Aber welche Kenntlichmachung, und wenn es mehr war, welche Veränderung hatte sich hier vollzogen! War in Paare Passanten immer noch von "Heimat" die Rede gewesen, die bei der Lektüre der Minima Moralia aufkomme, so wird hier, in Niemand anderes, nur noch von "gesellschaftskritischer Überheblichkeit” gesprochen. "Grausam, totalitär" sei ein Gedankengang der Minima Moralia, gar ein "großer Geistesschaden". Worum geht es dabei? Die Reflexionen aus dem beschädigten Leben werden jetzt angegriffen, weil sie selbst zur Beschädigung des Lebens beitragen. Die totale Kritik eines jeden Aspekts des Lebens führt, so Strauß, in letzter Konsequenz zu Lähmung und Erstarrung. Die Kritik will dem Leben in dieser Welt einen immanenten Sinn absprechen. Es geht Strauß also um die moralische Verurteilung eines Gegners im Namen des Lebens (vgl. SUTER 2001: 61-71). Er selbst vertritt dagegen einen absoluten Positivismus - nicht als Austreibung des Geistes, sondern als Aufforderung, sich völlig zu inkarnieren: Dieses Leben und nicht ein früheres oder zukünftiges soll als Aufgabe betrachtet werden. Was hier und jetzt ist, muss gelten und zum Bewährungsgrund menschlicher Wünsche und Absichten werden.

Botho Strauß sieht die "totalitäre" Kritik als ein Kind der Aufklärung. Diese ist allerdings nicht nur als eine bestimmte Epoche der Geistesgeschichte zu verstehen. Zwar wendet er sich, wie im Falle von Voltaire, auch deutlich gegen die historische Aufklärung, aber die eigentliche Kritik gilt einer Aufklärung, die meint, mit vernünftigen Mitteln Verfall und Untergang der Menschheit nachweisen zu können - ohne sich selbst in Irrationalität und Mythos zu verstricken:

Das Ende bleibt immer ein Mythologem. Und stets ist es total und einmalig im Horizont seiner Gläubigen, ganz gleich, ob es als Naturkatastrophe, Götterdämmerung, Weltbildsturz oder Terracid auftaucht. Die Kraft der Tatsächlichkeit ändert kaum etwas an der uralten Stimmung. Enden lässt sich nicht restlos säkularisieren. Auch nicht mit den letzten Finessen der aufgeklärten Vernunft. [...] Die Christen sind des Anfangs nicht kundig; sie sind seiner vergesslich, sie nehmen die Schöpfungsgeschichte nicht ernst genug. Besessen sind sie dagegen vom Ende in Heils- und Unheilsgeschichte. Nicht von ungefähr sind es ihre eschatologischen Epigonen, die Marxisten, aus deren gebrochenen Vernünften, gescheiterten Hoffnungen jetzt die unheilsgeschichtlichen Dämpfe und Ahnungen am stärksten entweichen. Der systematische Pessimismus als Folge der Aufdeckung 
Wefelmeyer, F. Die Sehnsucht nach Transzendenz

einer systematischen Geschichtstäuschung - aber was sind schon helle Köpfe wert, die einen Kater haben? (STRAUß 1987: 133; vgl. auch SCHAUBERGER 2000: 247)

Von Gebrochenheit und Finessen der aufgeklärten Vernunft spricht Strauß, an anderer Stelle ist von der "Roheit der Vernunft" die Rede, die den "grausamen Tatsächlichkeiten", die sie kritisiert, vom Wesen her verwandt sei: Weshalb Strauß ja auch einen Gedankengang der Minima Moralia "grausam" nennt. Es ist dies die Vernunft einer linken Intelligenz, die die Gegenwart als Katastrophe und Totalentfremdung analysiert und den lebenden Menschen als Hoffnung nur ein gesellschaftliches Jenseits anbietet. In dieser linken Vernunft mischen sich, laut Strauß, Motive der Gesellschaftskritik mit solchen der Theologie. Die Hoffnung auf Erlösung wird hier säkularisiert. Strauß dürfte dabei ebenso an Adornos Negative Dialektik wie an Blochs Das Prinzip Hoffnung und Atheismus im Christentum gedacht haben. Seine eigene Radikalkur will sich von linken Geschichtsphilosophien absetzen: "Das Jenseits zurück an seinen Platz. Und wenn Du unter Menschen gehst, sieh länger hin und hoffe kürzer" (STRAUß 1987: 202).

Ob sich Strauß mit dieser Kritik in das ideologische Lager der Gegenaufklärung begibt, bleibt zu diskutieren (vgl. WIESBERG 2002). Die Erörterung der Konsequenzen für sein Werk und seine politische Position hat mittlerweile eine umfangreiche Forschungsliteratur anwachsen lassen (HAVERTZ 2008: Bde 1 und 2). Klar ist aber, dass Strauß in der linken Gesellschaftskritik eine falsche Antwort auf die Sehnsucht nach Transzendenz sah und weiterhin sieht. Dieser Einsicht, wie kaum eine andere, wird später auch die Kritik an anderen Weltbildern, z. B. bestimmter christlicher Auffassungen, anregen. Sie treibt aber auch die weitere Entwicklung seines eigenen Schreibens in eine neue, bis jetzt geltende Richtung. Eine anhaltende Suchbewegung setzt ein, die der Sehnsucht nach Transzendenz mit anderen Denkmitteln und Anschauungen gerecht werden will. Sie führt bald auf die Wieder- und Neuentdeckung mythischer Weltbezüge und die zeitgenössische Literaturkritik hat dies auch notiert (vgl. BERKA 1991: 13-5).

Diese Entwicklung verläuft parallel zu Strauß' Versuchen, den linken Geschichtsphilosophien eine Konzeption der Geschichte entgegenzusetzen, die deutlich Anleihen bei der Chaosforschung, der Theorie des Big Bang, der Kybernetik und 
Wefelmeyer, F. Die Sehnsucht nach Transzendenz

Informationstheorie, und dem evolutionären Konstruktivismus macht. Das Ende der Geschichte, so argumentiert er, ist offen, die Entwicklung der Materie ist nicht determiniert. Aber die vom Geist geschaffenen Strukturen erzwingen auf jeder neuen Stufe ihrer Entwicklung eine andere Form menschlicher "Angemessenheit" im Umgang mit diesen Strukturen (STRAUß 1987: 135). Diese Situation verlangt zunächst einmal, dass der Forscher genau wahrnimmt, was eigentlich im Augenblick vorliegt:

Alles, was überhaupt ist, begreift er aus einer Ausschüttung, einem [...] sich strecken und entfernen. Er lässt auch dem jeweils Bestehenden, das er genau betrachtet, eine größere Gerechtigkeit widerfahren als der negative Utopist, der kaum genauer hinsieht, sondern mit augenzwinkerndem Verstand zu bedeuten gibt, dass alles übel ist und seiner gesellschaftlichen Erlösung harrt. (STRAUß 1987: 133)

Das Bestehende sieht Strauß aber vor allem als den Zusammenhang zwischen der natürlichen Umwelt und der vom Geist geschaffenen hochkomplexen Technologie. Die gesellschaftlichen Normen und Institutionen müssen dieser Konstellation entsprechen: In ihr sich bewegen zu können, ist die Aufgabe des Menschen. Gesellschaft an sich, als soziologisches Phänomen, verliert dagegen für Botho Strauß an Bedeutung. Gesellschaftstheorie, das Schibboleth linker Intelligenz, spielt in den jüngeren Werken von Strauß kaum eine Rolle. Lediglich das Werk des konservativen Soziologen Arnold Gehlen scheint einen nennenswerten Einfluss zu haben, eben weil es den Zusammenhang von Technik und Gesellschaft thematisiert (vgl. Strauß 1987: 144).

Von Gesellschaftstheorie und Kulturkritik erwartet Strauß keine Richtlinien, wie die modernere Technologie beherrscht werden kann. Die Technologie ist ein geistiges Produkt, in dem der Geist oder das Bewusstsein seine eigenen Strukturen vergegenständlicht hat: Computerisierte Informationssysteme sind zum Beispiel dem "neuronalen Schaltwerk des Gehirns" nachgebildet. Die Aufgabe des Geistes ist es jetzt, Meister dieses Produkts zu bleiben. Zu diesem Zweck muss er "Technosophie" entwickeln:

Zuviel Hirn, zuviel Umriss von Bewusstsein ist in die Dinge getreten und in unsere Hände geraten. Wir können sie nie wieder allein lassen. Der Geist, um mehr als ihr Wärter, nämlich ihr Meister zu sein, wird technischer und metaphysischer zugleich werden. Nicht im Widerstand gegen sein technisches Zeug, sondern in Koevolution mit ihm wird er seine Souveränität behaupten. Nicht die Höllenphantasmagorie des 
Kulturkritikers, sondern die Weisheit des Technikers empfinge uns dann am Ende des langen Wandels. Dort, nahe am Wunder, Technosophie (Strauß 1987: 135).

Nur wer den komplexen Zusammenhang zwischen geistiger Schöpfung und der in der Technik sichtbaren Außenverlegung mentaler Strukturen und Fertigkeiten erkennt, nur wer Natur als integralen Bestandteil eines die Fähigkeiten des Menschen einschließenden und sich mit ihm entwickelnden Systems versteht, "im Biotop einer Sumpfweise wie im hochintegrierten Halbleiterkreis", kann sich zugleich "abhängig darin und davon bewegen, ohne den größten Schaden anzurichten, das wird die nächste Anstrengung des Begriffs, die wir zu leisten haben" (ebd).

Es bleibt allerdings bei Strauß unausgeführt, wie der Mensch eigentlich die Geister, die er gerufen hat - um ein Bild aus Goethes Zauberlehrling hier anzuwenden -, auch beherrschen kann. Strauß entwirft ein Bild der Technik, in dem diese zwar vom Menschen entworfen ist, aber dann eine Entwicklung nimmt, die den Menschen, zumindest dem Tone nach, in dem Strauß schreibt, nicht zum Verfüger über die Technik macht, sondern ihn in ein Verhältnis der Zwangskooperation bringt. Es scheint, als ob mit einem Male auch die Technik ihr eigenes Sagen hat. Ihre Meinung ist sozusagen vom Menschen stets einzuholen, wenn er seine eigene Zukunft gestalten will. Der Gedanke einer möglichen Idolatrie des Technikwesens und der dann notwendigen Selbstbehauptung des Menschlichen gegenüber der Technik taucht zwar bei Strauß auf und wird auch zunehmend behandelt, aber ihm wird keine grundlegende Bedeutung gewährt, da Technik als geistiges Produkt des Menschen gesehen wird und daher im Prinzip menschlich ist. Missbrauch der Technik, zum Beipiel in der Neurotechnologie, liegt in den unerfüllten Sehnsüchten des Menschen begraben, die Lösung ist also auf Seiten des Menschen zu suchen (STRAUß 1992: 89).

Natürlich kennt Strauß das Argument, das vor allem von der frühen Frankfurter Schule entwickelt worden ist, dass in der Technik der Mensch sich entfremdet gegenübertritt und das eigene Produkt nicht als eigenes, sondern als zweite Natur, also als Naturzwang, begreift. Es bleibt aber fraglich, ob Strauß wirklich die geistige Natur des Menschen im Unterschied zur Technik begreift, wenn er diese Entfremdung dadurch 
Wefelmeyer, F. Die Sehnsucht nach Transzendenz

aufheben will, dass er die Technik selbst als Schöpfungsvorgang verstanden wissen will, eben zum Beispiel als Außenverlagerung neuraler Gehirnschaltungen. Demgegenüber ist meines Erachtens hervorzuheben, dass die Technik lediglich die automatisierte und mechanisierte Form jener Handlungsabläufe ist, mit denen wir die physischen Voraussetzungen schaffen, um Intentionen $\mathrm{zu}$ verfolgen, Wünsche $\mathrm{zu}$ verwirklichen und Bedürfnisse zu befriedigen (BALLHAUSEN 2010: 15-44).

Vom Gebrauch des Hammers bis zur Informationverarbeitung durch den Computer, immer geht es darum, menschliche Handlungen zu ersetzen, die zweckrational gebunden sind. Ist das einmal geschehen, werden Kraft und Zeit frei, sich handelnd auf anderes einzulassen. Der Schöpfungsvorgang ist geronnen in eine technische Handlungsform. Diese steht am Ende eines Prozesses, dessen Anfang durch die durch keine Technik zu erledigende Tatsache begründet ist, dass der Mensch seiner geistigen Selbstbestimmung nach entscheidet, was er will und wie er es handelnd ausführen will. Die Technik nimmt dem Menschen weder die Intentionen noch die Wünsche noch die Bedürfnisse ab. Sie selber verdankt ihre Existenz, meiner Auffassung nach, der im menschlichen Geiste geschöpften und vom menschlichen Willen realisierten Gestalt, die als originelle Schöpfung nur dem Menschen zugänglich ist. Selbst da, wo wir Formen höherstufiger Technik entwickelt haben, zum Beispiel informationsverarbeitende Maschinen, die den Erfolg ihrer eigenen Verarbeitung durch neue Informationsaufnahme kontrollieren, sind wir nicht mit einem neuen Schöpfungsvorgang konfrontiert, sondern bloß mit nach Prinzipien geregelten Handlungsformen. Die Prinzipien selbst haben einen einmaligen Schöpfungsort und -zeitpunkt, der im menschlichen Geist liegt. Was danach folgt ist nicht Schöpfung, sondern Wiederholung des schon bekannten Prinzips.

Eine solche Klarstellung ist hier meiner Ansicht nach notwendig, um den von Strauß gebrauchten Geist- und Schöpfungsbegriff genauer zu beurteilen. Es scheint nämlich, dass Strauß sich nicht völlig vom mechanistischen Naturbild der Naturwissenschaften des 18. und 19. Jahrhunderts befreit hat - trotz gegenteiliger Behauptungen. Möglicherweise ist er hier das Opfer einer Unschärfe geworden, die auch viele Vertreter jener neuen Wissenschaftszweige befallen hat, auf die Strauß über die 
Wefelmeyer, F. Die Sehnsucht nach Transzendenz

letzten 25 Jahre hin in mehreren Essaybänden mit Zustimmung verwiesen hat. Sei es in der Kybernetik, oder sei es in der Cognitive Science, man ist fasziniert von derselben Entdeckung: Biologische Strukturen des menschlichen Körpers, vom Blutkreislauf bis zum Nervensystem, weisen Organisationsformen auf, die in der natürlichen Außenwelt wiedergefunden werden können, die aber auch vom Menschen immer schon, ohne sein tieferes Wissen, handelnd in der Außenwelt oder vorstellungsmäßig in seiner inneren Erfahrungwelt wiederholt worden sind. Man spricht von mentalen Strukturen, Information oder auch Geist. Hierzu ein Beispiel aus Die Fehler des Kopisten:

Wer mit Maschinen arbeitet, bekommt ein Maschinenherz. (Chuang-tze) Doch wenn mein Herz das Vorbild der Maschine wird, die ich erbaue und betätige, so wird mir der Geist des Herzens begegnen. Er liefert die Blaupause, nach der auch das Gebilde meiner Hand, ohne zu leben, wie ein Organ funktioniert. Bios und Biotechnologie verstaendigen sich ohne Maschinenbegriff. (STRAUß 1997: 172)

Da der Geist aber immer an physisch wahrnehmbaren Formen erscheint, kommt es bei vielen Wissenschaftlern, aber eben auch, wie mir scheint, bei Botho Strauß, nicht zu einer wirklichen Erfahrung des lebendigen Geistes, sondern nur zu einem analytischen Erschließen oder modellhaften Konstruieren von Regelsystemen und mentalen Organisationsprinzipien, die im Gegenstand als wirkungsmächtig vorgestellt werden. Diesen Regeln und Prinzipien ist, nach dieser wissenschaftlichen Auffassung, auch der Mensch unterworfen. Er ist daher nicht der aktive Gestalter und Schöpfer des geistig Neuen, sondern das Objekt der Schöpfung. Der Schöpfungsakt selbst reduziert sich auf einen biologischen Vorgang, der sich am Menschen abspielt. Der Mensch ist hier ein, wie zugegeben wird, hochkomplexes geistiges System, aber eben nur ein System unter anderen geistigen Systemen. Sein Ich wird von Strauß deutlich in die Schranken verwiesen (STRAUß 1992: 11f.).

Da aber die systematisch relevante Erfahrung fehlt, dass am Ursprung jeder menschlichen Neuschöpfung die geistige Aktivität des Menschen steht, dass, mit anderen Worten, keine menschliche Neuschöpfung in die Welt tritt, es sei denn der Mensch habe sie aktiv hervorgebracht, da diese Erfahrung also fehlt, so fehlt, trotz der Vorstellung eines offenen Universums, auch eine Begriffsbestimmung der Freiheit bei Strauß (vgl. 
Wefelmeyer, F. Die Sehnsucht nach Transzendenz

SCALIGERO 1995: 9-82; BLUMENBERG 1998: 85-90). Stattdessen beobachten wir, wie bei Strauß, unter der Hand, wissenschaftliche Richtungen und Schulen wie zum Beispiel die des "evolutionären Konstruktivismus" zu den eigentlichen Handlungssubjekten werden:

\begin{abstract}
Niemand besitzt heute auch nur eine blasse Vorstellung davon, was dieser evolutionäre Konstruktivismus eigentlich sucht und will. Nichts geschieht hierbei nach einem zweckbestimmten Plan. Vielleicht steht gar am Ende aller Künstlichkeit der wahre >biologische< Mensch - das im Reich der Systeme mitversicherte Geschöpf, das sein gesamtes organisches Funktionieren nachgebildet und ins Äußere getragen hat [...] An die Stelle der Freiheit des Bewusstseins träte dann die vielbedingte Koexistenz von Werk und Geist, die Naturgesetzlichkeit von artifiziellem und biologischem Leben, die Systemharmonie von Organ und Apparat. (STRAUB 1987: 136)
\end{abstract}

Diese Eingrenzung des Schöpfungsbegriffs taucht, wie wir noch sehen werden, wieder auf, wenn man das Interesse verfolgt, mit dem Strauß versucht, der Kunst eine transzendente Aura zurückzugeben. Der in der abendländischen Tradition hergestellte Zusammenhang zwischen Schöpfungsakt und Kunstwerkproduktion, den die theologische Spekulation ja noch in Analogie zum göttlichen Schöpfungsakt der Welt gesehen hat, ist bei Strauß nicht völlig aus dem Bewusstsein verschwunden. Er macht aber von der Vorstellung keinen systematischen Gebrauch, dass sich die Einzigartigkeit eines Kunstwerkes aus der Fähigkeit des Künstlers ergeben könnte, nicht sklavisch Vorgegebenes reproduzieren zu müssen, sondern neu schöpfen zu können: Es ist die Fähigkeit, in die Welt eine sinnlich erfahrbare geistige Gestalt zu bringen, die früher im menschlichen Bewusstsein keine Existenz hatte.

Eine solche Vorstellung künstlerisch-schöpferischen Neubeginns braucht zu ihrer Stützung keine theologische Rechtfertigung, wiewohl man die Analogie zwischen künstlerischer und alttestamentarisch-göttlicher Schöpfung selbst nicht von der Hand weisen kann. Strauß macht sich aber, wie gesagt, diese Vorstellung nicht zu eigen, da die Idee eines Schöpfungsbeginns zur Auffassung eines linearen Geschichtsverlaufs und daher auch eines Endes der Geschichte führen würde. An dieses Ende hängen sich aber nach Strauß' Auffassung, wie schon ausgeführt, verderbliche Erwartungen, die die Gegenwart verneinen und letztlich auf Kosten des Lebens gehen. Strauß hat dagegen das 
Wefelmeyer, F. Die Sehnsucht nach Transzendenz

anspruchsvolle und in seinen Arbeiten noch weiterwirkende Programm der Beginnlosigkeit gesetzt (STRAUß 1992). Hier weitet er den Schöpfungsbegriff aus, macht ihn aber auch unspezifischer: Eigentlich ist alles Schöpfung, der gesamte Kosmos ist Schöpfung. Diese Schöpfung erscheint uns nur wie ein Chaos, tatsächlich ist sie, laut Strauß, von einer Ordnung, die so fein ist, dass wir sie bis jetzt noch nicht ausreichend verstanden haben. Dem Kosmos müssen wir uns aber verbinden, um die Fahrtrichtung des Raumschiffes Erde $\mathrm{zu}$ erkennen und die Einflussssphäre, der dieses Raumschiff untersteht, zu begreifen. Kosmologische Theorien, erhärtet durch Astro- und Teilchenphysik, lehren, so Strauß, dass sich die Welt im Prozess ständiger Schöpfung befindet.

Diesen rasanten Schöpfungsprozess erfährt der Mensch aber zunächst als "Raserei”, der er nur die Ruhe des Bewusstseins entgegensetzen kann: "Es gibt kaum ein erkennbares Ende der Materie. Das Elementare gleicht der Zwiebel des Peer Gynt - es besitzt keinen letzten Kern. Im Grunde herrseht das Grenzenlose in ewiger Beschleunigung" (STRAUß 1987: 140). Mit dem menschlichen Bewusstsein haben wir aber auch laut Strauß' Diagnose diejenige Instanz ausgemacht, die für das menschliche Unglück letztlich verantwortlich ist: nämlich die falsche Verabsolutierung des Menschen, die ihn außerhalb und überhalb der Schöpfung stehen lässt. Das Bewusstsein ist die Instanz der Negation, "Schöpfungsnichtung", und das heißt eben auch der negativen Gesellschaftstheorie, der negativen Kritik, der Unzufriedenheit mit dem Gegebenen. Wie das Bewusstsein letztlich dazu kommt, erklärt Strauß nicht oder nur in mythologischen, quasitheologischen Spekulationen. Wohl aber weiß er, wie dem Bewusstsein zu helfen ist: Es muss sich wieder in den Prozess der Schöpfung stellen, muss zu einer Bewahrungsform der Schöpfung werden, dabei aber selber schöpferisch sein! Das Bewusstsein darf nicht auf ein Ende der Schöpfung hoffen, also auf eine falsche Rückkehr ins Paradies, wo die Schöpfung alttestamentarischer Auffassung nach entstanden ist und abgeschlossen wurde (BOLLMANN 1989: 253-274). Und gerade weil er die Schöpfung als offenen Prozess denken will, fürchtet er eine technische Entwicklung, die zu Naturzerstörung, Selbstverlust und Hypertrophie des Individuellen und Privaten führt. So stellt er sich mit Grausen, zum Beispiel, die Möglichkeit vor, dass eine neue Technologie den Himmel zu einer riesigen 
Wefelmeyer, F. Die Sehnsucht nach Transzendenz

"Projektionsfläche" machen und dann jedem Zeitgenossen zur Verfügung stellen könnte: "Das Tohuwabohu des Privaten entstiege wie die Untoten der Erde, und bemächtigte sich des blanken Azurs" (STRAUß 2009: 9-10). Gerade auch in seinen späteren Arbeiten hat Strauß den Missbrauch besonders digitaler Techniken im Leben des Individuums beschrieben (PETER 2012: 175-185).

\section{Unglückliches Bewusstsein des Individuums und Transzendenz}

Kommen wir jetzt zur zweiten Form der Sehnsucht nach Transzendenz. Es ist die Sehnsucht des entmachteten und seiner selbst nicht mehr mächtigen Individuums, die aber, wie bereits gesagt, nur im Vorübergehen behandelt werden soll. Der theoretischsoziologisch aufbereiteten Unzufriedenheit, die in der Gegenwart nur Entfremdung und kapitalistische Ausbeutung sieht und daher auf das richtige Leben noch wartet, korrespondiert laut Strauß eine bestimmte individuelle Gefühlslage und intellektuelle Benommenheit. Das Individuum ist dieser Diagnose nach mit sich und der Welt völlig zerfallen, andererseits aber auch wieder bis zur Unkenntlichkeit mit der gesellschaftlichen Welt zur Deckung gebracht. Ewiges Nörgeln, Klagen und Wehleidigkeit stehen auf der einen Seite, dreistes Nachplappern der medialen Redeangebote, widerstandsloses und stromlinien-förmiges Mitschwingen auf den Ätherwellen des öffentlichen Bewusstseins, so lustbetont wie kopflos, stehen auf der anderen. Das Individuum ist auf beiden Seiten zu Hause, die tragende, ausgleichende und versöhnende Mitte fehlt aber. Und statt Halt zu geben, bleiben soziale Beziehungen in den elektronischen Netzwerken und DatingAgenturen hängen.

Stattdessen wechselt, so Strauß, das Eintagsbewusstsein seine Aggregatzustände, wie es sich eben ergibt. Mal krittelndes Denken und narzisstisch übermalte Totalkritik, mal affiges Showing-off von Status, Einkommen, Partner, Lebensversicherung, politischem Engagement oder Dritthaus in der Toskana. Dazu gehört natürlich auch die Angeberei mit dem eigenen Körper, sei er nun durch hartes Bodybuilding gestylt oder im Sonnenstudio angebräunt oder durch Müsli, Vollwertkost und Akupunktur gegen Krebskrankheit resistent 
Wefelmeyer, F. Die Sehnsucht nach Transzendenz

gemacht. Oder auch einfach alles. Wie eben im Fall derjenigen Frau in einem von Strauß' Stücken, die auf die Frage nach ihren Interessen antwortet: "Was mich interessiert? Meine Fantasie. Meine Beine. Meine Einheit.” (STRAUß 1986: 38) Lakonischer, gleichgültiger sagt es eine andere: "Na ja. Nun ist es eben so gelaufen. Voriges Jahr hatte ich in Keitum ein Lädchen im Kunsthandwerk. Dieses Jahr hab ich mir mal nichts vorgenommen... Ich hätte ja auch ein Kind bekommen können” (STRAUß 1982: 222).

Letzteres Zitat stammt übrigens aus einem der früheren Stücke. An der Diagnose, am Befund hat sich seitdem nichts geändert, auch wenn mittlerweile die Dynamik der Verhältnisse, nicht zuletzt wegen der weltweiten ökonomischen Krise, im Stau des Schlangestehens ihr bestes Bild gefunden hat: "Vor einem Einlass sich stauende Menge Flugzeug, Stadion, Apple Store, Essensausgabe. So ließe sich der Haufe immer derselben Gedrängten auf dem Wege vom Luxus zur Not verfolgen” (STRAUß 2013: 9-10.). Man könnte allerdings sagen, dass die Beschreibung genauer und deutlicher geworden ist. Der totalen Vordergründigkeit, mit der das Individuum in Strauß' früheren Theaterstücken sich und die Welt beschreibt, korrespondiert jetzt eine, paradox formuliert, vertiefte Darstellung jener Leerstellen im Bewusstsein, die einst transzendente Mächte besetzt hielten. Diese Mächte gaben früher dem Individuum Form und Orientierung. Vielleicht hat dies etwas mit der Erweiterung des Genrerepertoires zu tun, die sich in Strauß' Werk vollzogen hat. Neben das Bühnenstück ist seit den achtziger Jahren das Prosawerk (und das Gedicht) getreten, das, in Erzählungen und Reflexionen, durch Literaturverweise und Detailuntersuchungen, die dramatische Arbeit ergänzt und weiterführt. Es wird uns nicht mehr nur die Abwesenheit von Transzendenz vorgeführt, der Leser kann nun auch genauer studieren, welche Formen die Sehnsucht annimmt, die das alltägliche Leben übersteigen, ihm Glanz und Größe geben möchten, mit anderen Worten, die Leerstellen ausfüllen sollen.

Stellvertretend mag hier eine Studie zu einer verunglückten Liebesgeschichte angeführt werden, die wie im Vorübergehen auch auf Shakespeares Sonette Bezug nimmt. Eine junge Frau schreibt ihrem ehemaligen Partner einen Brief zum Hochzeitstag mit der neuen, jungen Frau: 
Da ist nichts, weshalb ich dich hassen müsste. Aber es gibt diese tiefe Verwundung, einem Mann so angehört zu haben und kein Kind von ihm zu besitzen. Das wirst du nicht verstehen. Aber ich, Freund, habe dir mein Leben gegeben und habe jetzt nichts, nichts in den Armen. Ich habe kein Leben von dir bekommen, keines, das über das meine hinauswachsen würde, das sich von selbst aufrichtete und mich auf eine erfüllte Weise überflüssig machte. [...] Dir mag es wünschenswert erscheinen und bequem, dass wir zu abgeklärten Menschen werden, die sich eines Tages wiedertreffen wie zwei Veteranen einer Liebesschlacht, solche, die sich gegenseitig die Ehrennadel der Tapferkeit und des Verzeihens an die Brust stecken. Zwei glücklich Davongekommene, die sich einst durch Himmel und Hölle jagten, und jetzt sitzen sie friedlich in deinem Garten, die Libelle des Rasensprengers kreist träg auf der Stelle, und ich spiele mit deinen Kindern. (STRAUß 1987: 27-33)

Gerade bei solchen Geschichten ließe sich vielleicht auch eine enge Verbindung zwischen den oben behandelten intellektuellen Theorien, die der Gegenwart nichts abgewinnen können, und dem unglücklichen Bewusstsein des einzelnen herstellen: Beide leiden an einem Übermass an Aufgeklärtheit und Rationalität, selbst da, wo sie sich gelegentlich in alternative Kulturen flüchten oder die Ablenk-Manöver der Spaßgesellschaft akzeptieren. Literatur, wie Strauß sie versteht, hält ihnen allen einen Spiegel vor, der zeigt, wie weit das Leben bereits zurückgestuft und abgeflacht ist: "Wie gut ließe es sich mit dem liebenswürdig verkommenen Tschechows leben! Doch die Verkommenen heute sind auf bösartige Weise unverträumt, aufgeklärt, vollkommen unsentimental. Durch und durch Gedämpfte. Problem-Knechte. Verstandesruinen” (STRAuß 1997:16). 
Wefelmeyer, F. Die Sehnsucht nach Transzendenz

\section{Realpräsenz im Kunstwerk und schöpferische Aktivität}

Es kann nicht verwundern, dass derjenige, der bei seinen Zeitgenossen die verzweifelten Wünsche nach Transzendenz unter die Lupe nimmt, selber von der Frage berührt wird, was am Ende der ausgespielten Computerspiele, der ausgeredeten Talkshows, der ausgeträumten Affären, was nach Partnertausch und Abenteuerurlaub eigentlich noch bleibt. Wenn die einst vom Autor selbst unterstützten Ideologien tot, wenn Fernseher und PC abgeschaltet und wenn die am Bewusstsein zappelnden Mitmenschen präzise und treffend beschrieben sind, muss auch der Schriftsteller seine "transzendentale Behausung" suchen, um hier ein Wort des frühen Lukács zu variieren. Strauß vertraut auf die kleine Gruppe, die den medialen Konsum verweigert und sich der herrschenden Geredekultur widersetzt. Zur Not ist diese Gruppe eine Einpersonengruppe, ihr Prototyp der Esoteriker:

Der Geheime ist heute schon der einzige Ketzer, der einzige wahrhaft Oppositionelle gegenüber der allesdurchdringenden, allesmäßigenden Öffentlichkeit. Gegen den totalen Medienverbund, gegen die Übermacht des Gleichgültigen wird und muss sich eine Geheimkultur der versprengten Zirkel, der sympathischen Logen und eingeweihten Minderheiten entwickeln. Kunst und schönes Wissen werden die Kraft der Verborgenheit [...] benötigen, um fortzubestehen und der verrückten, tödlichen Vermischung zu entgehen. Was sonst noch ist, gehört den Gewitzten und Amüsierten. (STRAUß 1986)

In seinem Buch Der Widerstand gegen die sekundäre Welt, das an Georg Steiners Idee der Real Presences anknüpft, wird Strauß dann noch genauer (STEINER 2010; STRAUß 2012). Dieser Text enthält ein sprachtheologisches Credo, er hat Bekenntnischarakter. Gleichzeitig ist er ein merkwürdiger Gedankenfrachter, offensichtlich um Tiefgang bemüht und angestrengt bedeutungsschwer. Wie anders liest sich der Autor in seinen Stücken, wie witzig und geistreich, wie amüsant und unterhaltsam schreibt er dort, dabei nie ohne tiefere Reflexion und stets um innovative Behandlung der Form bemüht. Hinter seinen Figuren sucht er das Versteck; Belehrung und Anweisung bleiben aus. Doch dieser Text lässt vor allem spüren, dass hier der Autor selbst spricht, dass er seiner Zeit den Weg aus der Misere weisen will. Insofern mag ihm die Rolle eines Schlüsseltextes zukommen. Allerdings 
Wefelmeyer, F. Die Sehnsucht nach Transzendenz

kommt er zunächst in pompöser Gestalt daher, mit viel Prätention. Das darf man nicht übersehen, muss man an betreffender Stelle kritisieren.

Da ist, ganz ernsthaft, von emergenten Summen, von Synchronisationen, von sich überlagernden Frequenzen, Vibrationen des Ursprünglichen, Fulguristen und revelatorischer Befreiung die Rede (STRAUß 2012: 37). Man hört zunächst, um wieder in Strauß' eigener physiognomischer Sprache zu reden, ein merkwürdiges Kauderwelsch aus Scientific American und Gruppendynamik. Doch Strauß bleibt nicht dabei stehen: Das als argumentativer Text verfasste Credo bietet einen diskutierbaren Kern an. Strauß möchte das Kunstwerk wieder mit der geistigen Welt verbinden. Der Kunst soll die mystische Aura zurückgegeben werden und in dieser sollen sich die heilenden Kräfte sammeln. Nichts darf sich deshalb zwischen das Kunstwerk und die sich in ihm verkörpernde Welt eines Göttlich-Geistigen drängen. Das Kunstwerk stellt nämlich den primären Bezug zum transzendent Göttlichen her, darum muss die sekundäre Welt der modernen Medien, aber ebenso auch die den Text auflösenden Interpretationsverfahren, wie sie zum Beispiel der Poststrukturalismus anbietet, ausgespart bleiben. Nur Text und Leser sollen sich gegenüber stehen. Das Leben aus zweiter Hand, eben das bloß kommentierende Gerede und die Simulation von Erfahrung, die die Medien bieten, würden das Berührtsein durch Kunstwerke verhindern. Die Interpretationsagenturen der Öffentlichkeit, so Strauß, würden sich wie Bakterien in den Text einnisten. Sie wollen alles zerreden und verhindern dabei, das der Geist des Lesers den Text innerlich bewegt und durch ihn bewegt wird.

Wo aber in den schönen Künsten die Erfahrung von Sinn gemacht wird, da geht es im letzten um einen Sinn, der auf die reale Gegenwart des Logos-Gottes verweist. Mit dem Begriff des Logos-Gottes knüpft Strauß, oft ohne die Quellen genauer zu nennen, an eine alte, aber dem Leser wohl kaum bekannte Tradition an (vgl. KELBER 1986). Im Kunstwerk ist der "Logos-Gott" anwesend, nicht weil das Werk ihn vertritt, sondern ihn mit jeder Form, mit jeder Zeile enthüllt. Strauß verdeutlicht das an der Abendmahlfeier:

Der geweihte Priester wandelt Weizenbrot und Rebenwein in die Substanz des Leibs und des Bluts Christi. Damit hört die Substanz der beiden Nahrungselemente auf, und nur ihre äußeren Formen bleiben. Im Gegensatz zur rationalen Sprachtheorie ersetzt das eine (das Zeichen, das Brot) nicht das fehlende andere 
Wefelmeyer, F. Die Sehnsucht nach Transzendenz

(den realen Leib), sondern übernimmt seine Andersheit. Dementsprehend müsste es in einer sakralen Poetik heißen: Das Wort Baum ist der Baum, da jedes Wort wesensmäßig Gottes Wort ist und es mithin keinen pneumatischen Unterschied zwischen dem Schöpfer des Wortes und dem Schöpfer des Dings geben kann. Gegenwärtig beim Abendmahl ist der reale Leib des Christus passus (d. i. im Zustand seines Todesopfers) unter der Gestalt des Brots. Das Gedenken im Sinne des Stiftungsbefehls ('Solches tuet aber zu meinem Gedächtnis.') wird dann zur Feier der Gleichzeitigkeit, es ist nicht gemeint ein Sich-Erinnern-An-Etwas. (STRAUß 2012: 40)

Das Kunstwerk ist hier also kein Bild, keine Metapher für die abwesende göttliche Schöpfung, sondern ein "Fenster”. Im Kunstwerk blicken wir auf die Gestalt des Schöpfers unter der Form des Kunstwerkes. Dies geschieht aber in großer Nähe zu unserem eigenen Wesen, denn wir selber sind sprachschöpferisch begabt und suchen, mit der Sprache Sinn, Harmonie, Ordnung und Schönheit zu schaffen und zu erfassen. Im Kunstwerk tritt uns ein Gleiches entgegen, dem wir aber zunächst unterlegen sind:

Die Unangemessenheit der sprachlichen Explikation, die Armut der Antwort, die wir auf die Fülle des Empfangs geben [...] ist eine erste Erfahrung des Unmittelbaren und der Andersheit, die im Kunstwerk Asyl genießen [...] Weder ist es ein utopisches Humanum noch ein höherer ästhetischer Gemütsreflex, noch überhaupt etwas vom Menschen Vermochtes, das sich in der Schönheit verbirgt. Vielmehr klingt in ihr an oder schimmert durch: Realpräsenz, Anwesenheit; und zwar unabhängig davon, welchen historischen oder biographischen Interessen sich die Entstehung eines Romans oder eines Gemäldes verdankt. Ob man einem Kunstwerk begegnet sei, meinte der metaphysisch nicht leicht erregbare Paul Valéry, erkenne man daran, ob es einen im Zustand der lnspiriertheit zurücklässt. Wir antworten mit Widerschein. (STRAUß 2012: 50f.)

Was zunächst auffällt ist, dass es offenbar bei Strauß keinen gedanklich klaren Übergang von den Schöpfungsmodellen der biologischen Wissenschaften zu der Annahme einer Realpräsenz des Göttlichen im Kunstwerk gibt. Die mögliche Wiederkehr eines Interesses an Realpräsenz wird zwar nach Analogie der Erklärungsweise verstanden, die die Wissenschaften geben, Stichwort: "Emergenz", aber in welchem Verhältnis die Wissenschaften zur Realpräsenz selbst stehen, bleibt unklar. Man darf vermuten, dass Strauß die menschliche Geschichte als Teil jener Schöpfungsgeschichte versteht, die Gegenstand der biologisch-kognitv orientierten Wissenschaften ist. Da er im Anschluss an George Steiner die Realpräsenz des Göttlichen an die sprachschöpferische Fähigkeit des 
Wefelmeyer, F. Die Sehnsucht nach Transzendenz

Menschen knüpft, ist es erstaunlich, dass die sprachschöpferische Leistung des Menschen, die sich in den Wissenschaften zeigt, nicht auch zu einer Präsenz des Göttlichen führt: Warum sollen Kunstwerke privilegierten Zugang zum Göttlichen haben? Offenbar fehlen Strauß und wohl auch Steiner die Mittel, Präsenzformen und Wissensformen als die beiden Seiten einer Sache zu sehen, so wie das etwa Novalis in seinem Programm einer Poetisierung oder Romantisierung der Naturwissenschaften getan hat. Anders gesagt, die schöpferischen Leistungen der Wissenschaften kommen so wenig in den Blick wie die Wissens- und Erkenntnisformen des Kunstwerks. Noch anders: Weder in den Wissenschaften noch im Kunstwerk wird etwas gewusst oder kommt etwas zur Erscheinung ohne den aktiv schöpferischen Mitvollzug des Menschen. Das scheint Strauß nicht wahrzunehmen oder, auf jeden Fall, nicht vorrangig wahrzunehmen.

Strauß konzentriert sich demgegenüber auf den Handlungvollzug, mit dem Präsenz erzeugt wird. Nicht zufällig ist ja auch die kultische Handlung des Priesters das Modell, an dem die Entstehung von Realpräsenz erläutert wird. Und es ist ja auch diese kultische Leistung des Künstlerpriesters, die die Unmittelbarkeit zum zuschauenden Leser herstellt und von der im zuletzt genannten Zitat bei Strauß die Rede ist. Vor den Augen des Lesers, in actu, vollzieht der Künstler die Präsentmachung. Die Lektüre verwandelt die Sehnsucht nach Transzendenz in die Erfahrung einer Realpräsenz dessen, was ansonsten alle Erfahrung übersteigt. Friedmar Apel hat zurecht darauf aufmerksam gemacht, dass dies die Befreiung der Sehnsucht ist (APEL 1999). Die Idee einer sozusagen liturgisch vermittelten Präsenz Gottes im Text bleibt aber an traditionelle metaphysische Vorstellungen gebunden: Die Essenz des Geistigen soll durch Schreib- und Leseakte in die Erscheinung gebracht werden. Deshalb werden auch die poststrukturalistischen Schulen mit Argwohn betrachtet, weil sie systematisch die Auflösung und das Auseinanderreißen von Schreib- und Leseakt betreiben.

Die Kritik an ihnen hat allerdings meines Erachtens etwas Kraftloses. Letztlich reduziert sie sich auf die einladende Geste, vom eigenen Geschäft abzulassen und den Text eben so zu lesen, wie es Strauß (und Steiner) wünschen. Die metaphysischen Annahmen verhindern am Ende ein Verständnis jener Fähigkeiten, die sich im Poststrukturalismus nur 
Wefelmeyer, F. Die Sehnsucht nach Transzendenz

von einer Seite, dann allerdings oft verabsolutiert, zeigen: dass der Leser nämlich wie der Künstler als geistige Wesen einen Neuanfang schaffen können, dass beide am Ende auf nichts anderes angewiesen sind und sich auf nichts anderes verlassen können, als auf ihre eigene geistige Aktivität. Transzendenz bestände dann darin, das Gegebene schöpferisch zu übersteigen, statt in ihm nur Ursache des eigenen Verhaltens zu sehen. Die moderne Physik, der Strauß zurecht großes Interesse entgegenbringt, sieht diesen Schöpferprozess in der Tat im ganzen Kosmos am Werke (vgl. DüRR 2010). In der Erfahrung von Kunstwerken kann dieser Prozess in ausgezeichneter Weise erlebt werden. Aber werden diejenigen, die sich dieser Erfahrung aussetzen, vielleicht bloß überwältigt und einem magischen Ritual oder Zwang unterworfen? Oder nimmt das Kunstwerk sie in Freiheit auf und entlässt sie in solcher auch wieder? Friedmar Apel hat in seiner Besprechung des Buches über Realpräsenz Strauß als Schamanen bezeichnet (APEL 1999). Ein Schamane braucht sich aber nicht unbedingt auf die geistige Selbständigeit und Aktivität seiner Klienten verlassen, viele Dinge können auch hinter deren Rücken erreicht werden. Wem aber würde dann das Kunstwerk wirklich dienen?

Strauß hat in seinem Werk auch den Gedanken der Kunst als Erinnerung des Mythischen stark gemacht: einer Erinnerung, die in die Präsenz gelangen soll. Möglicherweise gelingt es seinem Werk, und es wäre ihm als Verdienst anzurechnen, dem Leser ein deutlicheres Bewusstsein dafür zu geben, dass er seine Gegenwart leben muss und nicht als bloße Durchgangsstation für ein späteres besseres Leben betrachten und damit verraten darf. Sicher, was könnte dieses Gegenwärtigkeitsbewusstsein besser steigern als die Erfahrung einer Realpräsenz des Göttlichen, einer Epiphanie, hier und heute im Kunstwerk. Doch Strauß muss sich vorhalten lassen, dass die Gegenwart zur Zukunft hin offen ist: In jedem Leseakt, in jedem Schreibakt wird möglicherweise neue Essenz geschaffen, auf die, um in Strauß' Metaphorik zu bleiben, kein Fenster je geblickt hat. Dies freilich gilt im Künstlerischen so wie im Politischen und wie im Verhältnis zur Natur. Das gilt auch unter theologischen Gesichtspunkten: Wenn Strauß das Kunstwerk auf die Erlösungstat Christi warten lässt, zwischen "Kreuzestod und Sonntag der Auferstehung”, so darf er nicht vergessen, dass diese Tat nur wirklich werden kann durch die Aktivität, mit 
Wefelmeyer, F. Die Sehnsucht nach Transzendenz

der ein frei handelndes Wesen an seinem Nächsten die Erlösungstat wiederholt - und damit den Gott selbst erlöst. Dieses Wesen ist der Mensch.

\section{Literaturverzeichnis}

APEL, Friedmar. Die Befreiung der Sehnsucht: Die gesammelten Pamphlete des Schamanen Botho Strauß. In: Frankfurter Allgemeine Zeitung, 23/3/1999, 69.

BALLHAUSEN, Wolfram. Technikphilosophische Aspekte der Automatisierungstechnik. Frankfurt am Main/Berlin/Bern/Bruxelles/New York/Oxford/Wien: Lang, 2010.

BAUER, Christian. Sacrificium intellectus: Das Opfer des Verstandes in der Kunst von Karlheinz Stockhausen, Botho Strauß und Anselm Kiefer. München/Paderborn: Fink, 2008.

BERKA, Sigrid. Mythos-Theorie und Allegorik bei Botho Strauß. Wien: Passagen, 1991.

BLUMENBERG, Hans. Paradigmen zu einer Metaphorologie, Frankfurt am Main: Suhrkamp, 1998.

BOLLMANN, Stefan. Vom erhabenen zum komischen, vom geschichlichen zum kosmologischen Denken: Botho Strauß im Kontext. In: PRIES, C. (Hg). Das Erhabene: Zwischen Grenzerfahrung und Größenwahn. Weinheim: VCH, Acta Humaniora 1989: 253-274.

FUSS, Dorothee. "Bedürfnis nach Heil": Zu den ästhetischen Projekten von Peter Handke und Botho Strauß. Bielefeld: Aisthesis, 2001.

HAGE, Volker. Schreiben ist eine Séance. Begegnungen mit Botho Strauß. In: RADIX, Michael (Hg). Strauß Lesen. München/Wien: Hanser, 1987: 188-216.

HAVERTZ, Ralf. Der Anstoß: Botho Strauß’ Essay “Anschwellender Bockgesang” und die neue Rechte: Eine kritische Diskursanalyse. Berlin: Traktor Verlag, 2008.

KELBER, Wilhelm. Die Logoslehre: Von Aristoteles bis Origines. Frankfurt am Main: Fischer, 1986.

KLUGE, Alexander. Die Artisten in der Zirkuskuppel, ratlos. München: Piper, 1968.

NOWAK, Marcin. Das 'Leben in Scheidung': Conditio humana im Werk von Botho Strauß. Dresden: Neisse, 2006.

PETER, Otto. Kritk der Digitalisierung: Warner, Bedenkenträger, Angstmacher, Apokalyptiker. Frankfurt am Main/Berlin/Bern/Bruxelles/New York/Oxford/Wien: Lang 2012.

REUS, Sebastian. Unglückliches Bewusstsein: Denken ohne Dialektik bei Botho Strauß. Würzburg: Königshausen und Neumann, 2006.

RICHTER, Anja-Maria. Das Studium der Stille: Deutschsprachige Gegenwartsliteratur im Spannungsfeld von Gnostizismus, Philosophie und Mystik: Heinrich Böll, Botho Strauß, Peter Handke, Ralf Rothmann. Frankfurt am Main/Berlin/Bern/Bruxelles/New York/Oxford/Wien: Lang, 2010.

SAUTER, Günter. Politische Entropie: Denken zwischen Mauerfall und 11.September (Botho Strau $\beta$, Hans Magnus Enzensberger, Martin Walser, Peter Sloterdijk). Paderborn: Mentis, 2002.

SCALIGERO, Massimo. Traktat über das lebendige Denken: Ein Weg zur Überwindung der

Pandaemonium, São Paulo, v. 16, n. 21, Jun/2013, p. 1-24 www.fflch.usp.br/dlm/alemao/pandaemoniumgermanicum 
Wefelmeyer, F. Die Sehnsucht nach Transzendenz

abendländischen Philosophien, Stuttgart: Urachhaus, 1995.

SCHAUBERGER, Sebastian. Permanenz der Urbilder: Mythische und biblische Anspielungen bei Botho Strauß. Bielefeld: Inaugural-Dissertation (Universität Bielefeld), 2000.

STEINER, George. Von realer Gegenwart. Hat unser Sprechen Inhalt? München: Hanser, 2010.

STRAUSS, Botho. Paare Passanten. München/Wien: Hanser, 1981.

. Groß und Klein. München/Wien: Hanser, 1982.

. Die Fremdenführerin: Stück in zwei Akten. München/Wien: Hanser, 1986.

. Der Geheime. Über Dieter Sturm, Dramaturg an der Berliner Schaubühne. In: DIE ZEIT, 23/5/1986, 22.

. Niemand anderes. München: Wien, 1987.

- Versuch, die ästhetischen und politischen Ereignisse zusammenzudenken. Texte über Theater 1967-1986. Frankfurt am Main: Verlag der Autoren 1987a.

. Beginnlosigkeit: Reflexionen über Fleck und Linie. München/Wien: Hanser, 1992.

. Der Fehler des Kopisten. München/Wien: Hanser, 1997.

. Vom Aufenthalt. München/Wien: Hanser, 2009.

_. Der Aufstand gegen die sekundäre Welt: Aufsätze. München/Wien: Hanser, 2012.

. Die Fabeln von der Begegnung. München/Wien: Hanser, 2013.

Recebido em 20/03/2013

Aprovado em 30/04/2013

Pandaemonium, São Paulo, v. 16, n. 21, Jun/2013, p. 1-24 www.fflch.usp.br/dlm/alemao/pandaemoniumgermanicum 